\title{
Adult sublingual schwannoma with angioma-like features and foam cell vascular change
}

\author{
Sarah Taconet ${ }^{1,2}$, Philippe Gorphe ${ }^{3}$, Adriana Handra-Luca ${ }^{1,2}$ \\ ${ }^{1}$ Universite Paris Nord Sorbonne Cite, Bobigny, ${ }^{2}$ Service d'Anatomie Pathologique, APHP Avicenne, Bobigny, \\ ${ }^{3}$ Service Oto-Rhino-Laryngologie et Chirurgie Cervico-Faciale, APHP Avicenne, Bobigny, France \\ All authors contributed equally and should be considered as first author.
}

\begin{abstract}
Schwannomas are benign, slow-growing neoplasms of the neural sheaths. They occur rarely in a sublingual location; 9 cases have been reported to our knowledge. We aimed to report the case of a sublingual schwannoma with peculiar morphological features. A 23-year-old woman presented for a right sublingual mass, clinically diagnosed as a vascular malformation. A pleomorphic adenoma was suggested by cytology examination. The diagnosis of schwannoma was made on the resected specimen. The tumour, measuring $4.1 \mathrm{~cm}$, showed several angioma-like vessel agglomerates and macrophage thickening of the intratumour vessel wall. Tumour cells expressed diffusely S100 protein, as well as CD56 and podoplanin. In conclusion, we report the case of a sublingual schwannoma, of peculiar morphology, with presence of pseudo-angioma aggregates and foam cell thickening of the vessel wall, features which were of clinical relevance.
\end{abstract}

Key words: schwannoma, salivary gland, sublingual, differential diagnosis, angioma, macrophage, podoplanin/D2.40, NCAM/CD56, CD68.

\section{Introduction}

Schwannomas are benign slow-growing neoplasms of the neural sheaths. They occur rarely in a sublingual location; 9 cases have been reported to our knowledge $[1,3,6,7,9,10,12-15]$. In only one of the reported cases the schwannoma was intraglandular [10]. The diagnosis is made on the surgical specimen, after surgical resection, possibly due to their rarity in this setting and to nonspecific clinical complaints $[1,3,6,7,9,10,12-15]$. Similarly to schwannomas in other locations, tumour cells express the $\mathrm{S} 100$ protein, neuron-specific enolase, and vimentin [13].
We report a case of a sublingual schwannoma, peculiar by the presence of angioma-like vessel agglomerates and macrophage thickening of the intratumour vascular wall.

\section{Case report}

A 23-year-old woman presented with a 2-year history of a slow-growing mass of the right sublingual space, clinically diagnosed as a vascular malformation. Clinical examination revealed a firm, mobile mass. There was no adenopathy or nerve deficit. Familial and personal medical histories did not reveal any disease except concomitant cystitis. 
At ultrasound examination, there was a heterogeneous, well-limited and well-vascularised sublingual mass. On magnetic resonance imaging (MRI), the tumour was located in the anterior third of the mouth floor, in a sublingual location, pushing the submandibular gland and the mylohyoid muscle, and in contact with the right horizontal branch of the mandible. The tumour was heterogeneous with central zones of $\mathrm{T} 2$ hypersignal. A moderate heterogeneous increase occurred after gadolinium injection. Fine-needle aspiration cytology analysis showed non-specific microscopic aspects with abundant haemorrhage, eosinophilic material, lymphocytes and epithelial-like cells. The suggested diagnosis was pleomorphic adenoma. The tumour with the contacting right submandibular gland was surgically resected. Perioperatively, the tumour was well limited by a capsule, of firm consistency. The hypoglossal and lingual nerves did not show direct attachment to the tumour, and were preserved.

Macroscopically, the tumour was well limited, encapsulated and measured $4.1 \times 3.5 \times 3.5 \mathrm{~cm}$. At microscopic examination, this tumour consisted of proliferating spindle cells, with frequent dense cellular zones of palisading of Antoni A pattern, and rarely with epithelioid features (Fig. 1). Cells showed nuclear atypia with a conserved nuclear : cytoplasmic ratio. Vessels were numerous, focally densely packed in multiple clusters without interspersed tumour cells, with a pseudo-angioma pattern, randomly disposed within the tumour (Fig. 1). Most vessels were thin walled, of capillary type, large, medium and small sized or having a cavernomatous aspect. Several haemorrhagic zones were also observed. Hyaline vascular parietal change was rare, as were reactive lymphocytes. Several thin-walled vessels, mainly medium-sized vessels, showed thrombosis. Some of these vessels, as well as several non-thrombotic thin-walled medium- or small-sized vessels, contained layers of subendothelial foamy macrophages (Fig. 1). In the latter vessels, the endothelial lining was continuous, focally hyperplastic/regenerative. Small sheets of foamy macrophages were observed, less frequently between tumour cells, mainly in oedematous zones. These foamy cells did not contain haemosiderin pigment. Submandibular gland parenchyma was normal, without vascular abnormality, with several millimetric sebaceous foci. Salivary gland lobules were present in a pericapsular location. At 6 months post-operatively, the patient was well, without recurrence, other tumour or nerve deficit.

An immunohistochemical study on tumour tissue sections was performed with XT Benchmark and Benchmark Ventana automates (avidin-biotin complex method) with antibodies directed against the following proteins: calretinin (Dako, clone DAK Calret1, 1/100), CD31 (Dako, clone JC70A, 1/20), CD34 (Dako, QBEnd-10, dilution 1/25), CD56 (Novocastra, NCL-L-CD56-1B6, dilution 1/100), CD68 (Dako, clone PG-M1, dilution 1/50), podoplanin (Dako, clone D2-40, dilution 1/100), CD117 (Novocastra, NCL-LCD117, dilution 1/40), chromogranin (Dako, clone DAK-A3, dilution 1/200), cytokeratin AE1/3 (Dako, clone $A E 1 / A E 3$, dilution 1/100), desmin (Dako, clone D33, dilution 1/100), EMA (Dako, clone E29, dilution 1/50), neuron-specific enolase (Dako, clone BBS/NC/ VI-H14, dilution 1/400), S100 protein (Dako, polyclonal, dilution 1/1500), smooth muscle actin (Dako, clone 1A4, 1/400), and synaptophysin (Dako, clone SY38, dilution 1/20).

Tumour cells expressed the S100 protein (Fig. 1), neuron-specific enolase diffusely, as well as synaptophysin. We also observed focal, cytoplasmic tumour cell expression of podoplanin, CD56 and CD68. The CD31 was expressed in the endothelial lining of intratumour vessels (Fig. 1), which showed focally an irregular layer of smooth muscle actin positive cells. Desmin was expressed only in the wall of sparse, large vessels in the pericapsular tumour zone. Podoplanin was expressed very focally in the lining cells of a cavernomatous space. Subendothelial foamy macrophages, as well as macrophages in xanthomatous foci, strongly expressed CD68. They also expressed CD31, less intensely than endothelial cells (Fig. 1).

\section{Discussion}

We report the case of a schwannoma of rare location, sublingual, showing several morphological peculiarities of clinical relevance, this tumour being unusual due to the presence of angioma-like aggregates and subendothelial foamy macrophage accumulations.

Schwannomas are benign tumours, extremely rare in a sublingual location, 9 cases being reported since 1960 to our knowledge [1,3,6,7,9,10,12-15] (Table I). In the 8 reported cases with available data for review, there is a female gender prevalence of $5: 3$ (women : men). Patient's median age is 52 years, 

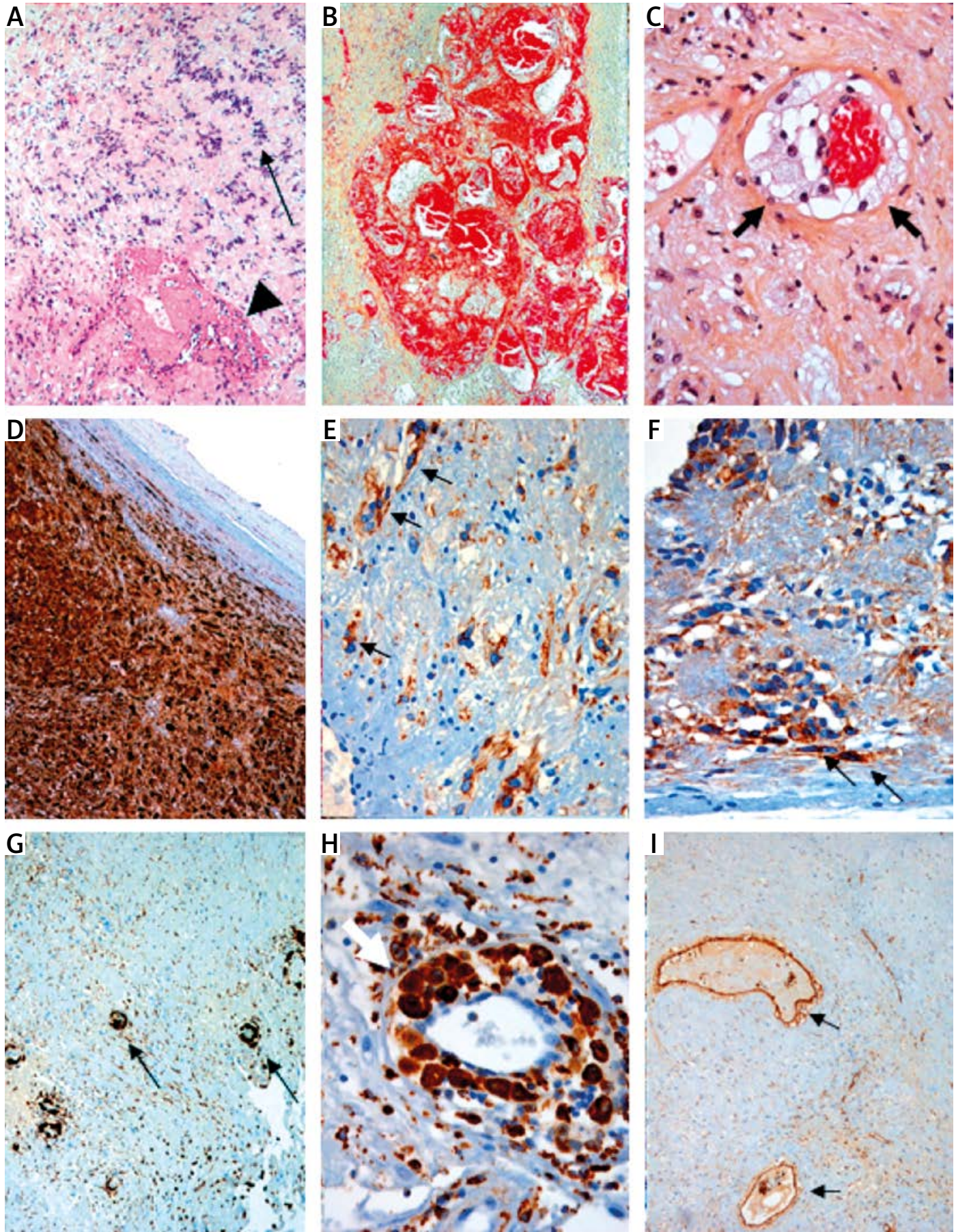

Fig. 1. Microscopic features of sublingual schwannoma. The tumour showed palisading Antoni A zones with spindle cells ( $\mathbf{A}-$ arrow, haematoxylin and eosin stain, original magnification $\times 10)$ and thrombosis in vascular cavities (A - arrowhead). Intratumour angioma-like vascular aggregates composed of several thin-walled vessels were also observed $(\mathbf{B}-$ haematoxylin and eosin stain, original magnification $\times 10)$. Tumour cells expressed diffusely the S100 protein $(\mathbf{D}$ - original magnification $\times 20)$ and focally CD56 (E - arrows, original magnification $\times 40)$ and podoplanin $(\mathbf{F}-$ arrows, original magnification $\times 40)$. Foamy macrophages $(\mathbf{C}-$ arrows, haematoxylin and eosin stain $\times 40), C D 68$ positive were observed in the vessel wall, in subendothelial location (G - arrows, original magnification $\times 20$, and $\mathrm{H}$, original magnification $\times 40$ ). Endothelial cells were strongly positive for CD31, which was also expressed by subendothelial macrophages, less intensely ( $\mathbf{I}$-arrows, original magnification $\times 10$ ). 
Table I. Reported cases of sublingual schwannomas

\begin{tabular}{|c|c|c|c|c|c|c|}
\hline Author, year & Age & Gender & Tumour size $(\mathrm{cm})$ & Nerve of origin & Cytology diagnosis & Peculiarity \\
\hline Okada, 2012 [10] & 70 & female & 2.3 & $\mathrm{NI}$ & & \\
\hline Pattani, 2010 [12] & 63 & male & 4.4 & $\begin{array}{l}\text { mylohyoid } \\
\text { nerve }\end{array}$ & $\begin{array}{c}\text { pleomorphic } \\
\text { adenoma }\end{array}$ & \\
\hline Kawakami, 2004 [6] & 49 & female & 3.2 & $\begin{array}{l}\text { lingual } \\
\text { nerve }\end{array}$ & & $\begin{array}{c}\text { cystic } \\
\text { degeneration }\end{array}$ \\
\hline Arda, 2003 [1] & 34 & female & 5 & $\begin{array}{c}\text { sublingual } \\
\text { nerve }\end{array}$ & inconclusive & \\
\hline Ogawa, 2001 [9] & 72 & female & 5.3 & $\begin{array}{l}\text { hypoglossal } \\
\text { nerve }\end{array}$ & & $\begin{array}{l}\text { multifocal } \\
\text { tumour }\end{array}$ \\
\hline Drevelengas, 1998 [3] & 55 & male & 4.5 & $\begin{array}{c}\text { hypoglossal } \\
\text { nerve }\end{array}$ & & \\
\hline Lee, 1994 [7] & 20 & female & 5 & $\mathrm{NI}$ & & \\
\hline Sengupta, 1975 [14] & NA & NA & NA & NA & & \\
\hline Swanson, 1960 [15] & 20 & male & 12 & $\mathrm{NI}$ & non-contributory & $\begin{array}{l}\text { multifocal } \\
\text { tumour }\end{array}$ \\
\hline
\end{tabular}

$N A$ - non-available, $N I$ - non-identifiable

ranging from 20 to 72 years. Only 2 patients were younger than 25 years, similarly to our patient. In the reported cases, the lesion was painless and treatment consisted of surgery. The diagnosis of schwannoma was made after the resection specimen analysis. The median tumour size was of $4.75 \mathrm{~cm}$, varying between 2.3 [10] and $12 \mathrm{~cm}$ [15]. Rarely tumours can be multifocal/multinodular $[10,15]$ The nerve of origin remains relatively frequently unidentified, in the reported sublingual schwannomas in 3 out of the 8 cases with available data (Table I). Similarly to the case reported by Pattani et al. [12], the nerve of origin in our case was probably the mylohyoid nerve, although we did not detect a direct connection to the tumour, as they did. In our case, this hypothesis was sustained by the lack of hypoglossal palsy, of dry mouth and change in salivary flow. Moreover, the hypoglossal and lingual nerves appeared normal at preoperative examination.

The presence of several intratumour angiomalike vessel aggregates along with the fibrous, oedematous change and haemorrhage were of clinical relevance, the tumour being initially diagnosed clinically as a vascular malformation. Moreover, presence of a prominent intratumour vascular component is related to an increased haemorrhagic risk at fine needle aspiration or operative manipulation [5]. The origin of such an intratumour vascular component is still debated as to whether it is of angioma or mal- formation type, part of a composite tumour or of angioma-like vascular change in the stroma [5].

Interestingly, in both our case and the sublingual schwannoma reported by Pattani et al. [12], the preoperative cytological features suggested a pleomorphic adenoma. The MRI features may also suggest a pleomorphic adenoma [7]. In all these cases of sublingual schwannoma, only the standard histological examination of the surgically resected tumour allowed the precise tumour type to be established and the schwannoma diagnosed.

The present case was unusual not only due to the presence of pseudoangiomatous vascular aggregates but also due to the presence of intratumour vascular lesions. These lesions consisted of an accumulation of subendothelial, CD68-positive, xanthomatous, foamy macrophages. Rarely such cells formed small sheets between tumour cells, described as related to long evolution of degenerative changes. To our knowledge, in the English language medical literature, presence of intratumour xanthomatous foci has been specifically reported in a few intracranial schwannomas as well as in two extracranial schwannomas, of perihepatic and thoracic locations $[2,8]$. Patients did not have a history of metabolic condition but had contrast substance injection for imaging procedures, similarly to our patient. The subendothelial accumulations of macrophages we have observed in our case are peculiar, but possibly belong to the same spectrum of such xanthomatous 
lesions. The hypothesis of a contrast-substance vascular reaction is sustained by the presence, in some of the vessels, of focal disruption and damage of the vascular endothelial lining with thrombosis, by the lack of personal or familial history of metabolic disease, and by the lack of similar deposits in the normal submandibular gland that we could examine. However, we cannot completely eliminate the possibility of degenerative changes related to a long evolution of the tumour or changes related to fine needle puncture. Abundant xanthomatous changes may result in intratumour heterogeneity on the CT scan, similarly to other schwannoma changes such as cellularity, cystic degeneration or collagen deposition [8].

Although eight cases of sublingual schwannomas have been reported, only three have been studied for S100 protein $[1,3,7]$, one of which has also been studied for neuron specific enolase, vimentin and Ki67 [1]. Tumour cells in our case expressed the S100 protein and neuron-specific enolase. Interestingly, we also observed focal tumour expression of CD56 and of podoplanin, both proteins having been already reported in schwannomas of other location [11]. While CD56 interferes with calcium-independent homophilic cell-cell binding [11], the role of podoplanin-positive schwannoma tumour cells remains incompletely understood, possibly interfering with occurrence of oedema. Indeed this protein has been reported to interfere with the regulation of inner ear fluid pressure of D2.40 positive periductal connective tissue cells [4].

In conclusion, we report a rare case of sublingual schwannoma, peculiar due to stromal angioma-like features, which might be relevant for clinical and imaging preoperative diagnosis.

\section{Acknowledgements}

We thank Isabelle Alexandre, Emilie Weber, Viviane Bodiguel, Emmanuel Sauce, and Didier Dunghi for technical and administrative support as well as Dr. Marcela Albiter, Dr. Claude Bigorne, Dr. Frederique Larousserie and Prof. Anne Couvelard for constructive discussion. The authors thank Pascal Cusenier for help with reference research.

\section{Disclosure}

Authors report no conflict of interest.
A new case of sublingual schwannoma occuring in an adult patient (Yamamoto et al.) was published since the acceptance of this manuscript.

\section{References}

1. Arda HN, Akdogan O, Arda N, Sarikaya Y. An unusual site for an intraoral schwannoma: A case report. Am J Otolaryngol 2003; 24: 348-350.

2. Cohen LM, Schwartz AM, Rockoff SD. Benign schwannomas: pathologic basis for CT inhomogeneities. AJR Am J Roentgenol 1986; 147: 141-143.

3. Drevelengas A, Kalaitzoglou I, Lazaridis N. Sublingual hypoglossal neurilemmoma. Case report. Aust Dent J 1998; 43: 311-314.

4. Hultgård-Ekwall AK, Mayerl C, Rubin K, Wick G, Rask-Andersen H. An interstitial network of podoplanin-expressing cells in the human endolymphatic duct. J Assoc Res Otolaryngol 2006; 7 : 38-47.

5. Kasantikul V, Netsky MG. Combined neurilemmoma and angioma. Tumor of ectomesenchyme and a source of bleeding. J Neurosurg 1979; 50: 81-87.

6. Kawakami R, Kaneko T, Kadoya M, Matsushita T, Fujinaga Y, Oguchi K, Kurashina K. Schwannoma in the sublingual space. Dentomaxillofac Radiol 2004; 33: 259-261.

7. Lee ES, Choi SC, Park TW, You DS. Schwannoma of the sublingual gland. Korean J Oral Maxillofacial Surg 1994; 24: 461-467.

8. Nagafuchi Y, Mitsuo H, Takeda S, Ohsato K, Tsuneyoshi M, Enjoji M. Benign schwannoma in the hepatoduodenal ligament: report of a case. Surg Today 1993; 23: 68-72.

9. Ogawa T, Kitagawa Y, Ogasawara T. A multifocal neurinoma of the hypoglossal nerve with motor paralysis confirmed by electromyography. Int J Oral Maxillofac Surg 2001; 30: 176-178.

10. Okada H, Tanaka S, Tajima H, Akimoto Y, Kaneda T, Yamamoto $H$. Schwannoma arising from the sublingual gland. Ann Diagn Pathol 2012; 16: 141-144.

11. Park JY, Park H, Park NJ, Park JS, Sung HJ, Lee SS. Use of calretinin, CD56, and CD34 for differential diagnosis of schwannoma and neurofibroma. Korean J Pathol 2011; 45: 30-35.

12. Pattani KM, Dowden K, Nathan CO. A unique case of a sublingual-space schwannoma arising from the mylohyoid nerve. Ear Nose Throat J 2010; 89: E31-33.

13. Rosai J. Rosai and Ackerman's Surgical Pathology. 10 ${ }^{\text {th }}$ ed. Elsevier Sanders Mosby, New York 2011.

14. Sengupta P, Sarkar SK, Niyogi SK. Sublingual neurilemmoma. J Indian Med Assoc 1975; 64: 238-240.

15. Swanson AE, White JB. The unusual occurrence of a large sublingual neurilemmoma. Oral Surg Oral Med Oral Pathol 1960; 13: $1163-1166$ 